Portland State University

PDXScholar

10-1-1998

\title{
A Note on the Green Dyadic Calculation of the Decay Rates for Admolecules at Multiple Planar Interfaces
}

\author{
P.T. Leung \\ Portland State University, hopl@pdx.edu \\ R. L. Hartman \\ Scott M. Cohen \\ Portland State University, scott.cohen@pdx.edu
}

Follow this and additional works at: https://pdxscholar.library.pdx.edu/phy_fac

Part of the Physics Commons

Let us know how access to this document benefits you.

\section{Citation Details}

Hartman, R. L., Cohen, S. M., \& Leung, P. T. (1999). A note on the green dyadic calculation of the decay rates for admolecules at multiple planar.. Journal Of Chemical Physics, 110(4), 2189.

This Article is brought to you for free and open access. It has been accepted for inclusion in Physics Faculty Publications and Presentations by an authorized administrator of PDXScholar. Please contact us if we can make this document more accessible: pdxscholar@pdx.edu. 


\title{
A note on the green dyadic calculation of the decay rates for admolecules at multiple planar interfaces
}

\author{
R. L. Hartman, S. M. Cohen, and P. T. Leung \\ Department of Physics, Portland State University, P.O. Box 751, Portland, Oregon 97207-0751
}

(Received 4 September 1998; accepted 23 October 1998)

\begin{abstract}
The Green dyadic formulation for calculating classical decay rates of admolecules at multiple planar interfaces first published by Chance, Prock and Silbey is reexamined. It is pointed out that, for the case of fluorescing molecules sandwiched between a system of super- and substrate interfaces, the original formalism requires significant modifications in order to lead to results consistent with those obtained from the Sommerfeld radiation theory. (C) 1999 American Institute of Physics.
\end{abstract}

[S0021-9606(99)70204-7]

\section{INTRODUCTION}

The study of molecular fluorescence at solid interfaces has been active for the last 2 decades since the first experimental works of $\mathrm{Kuhn}^{1}$ and Drexhage ${ }^{2}$ done in the early 1970's. Theoretically, it has been found that one of the most simple and direct approaches is to follow a phenomenological model solving the electrodynamics of an emitting molecular dipole in the vicinity of the interfaces. This model can provide both frequency shifts and decay rates for the admolecules (normalized to the free molecular decay rate), for example, yielding results in agreement with experimental results as well as quantum mechanical calculations. Among other contributors, Chance, Prock and Silbey (CPS) have cleverly applied the radiation antenna theory of Sommerfeld ${ }^{3}$ to this problem and showed that classical electrodynamics alone can account for most of the experimental observations. A momentous review article ${ }^{4}$ was compiled by CPS in the late 1970's summarizing the complete status of the subject at that time. Over the last 20 years, this article has often been quoted and used by people working in the field, experimentalists and theorists alike. ${ }^{5}$ Application of this theory has also gone beyond fluorescence to other optical phenomena at interfaces as in Ref. 6 and 7. It is also in this review article that the Sommerfeld method $^{3}$ was first generalized to the case of multiple planar interfaces using the dyadic Green's function formulation. This generalization includes both cases (i) where the layer of fluorescent dyes is deposited on the top of a stratified multilayer system and (ii) where the layer is sandwiched between two such multilayer systems.
It is the purpose of this paper to point out that, in the original CPS formulation for case (ii) above, the choice of the dyadic eigenfunctions was not appropriately made and significant modifications are necessary to obtain a consistent Green dyadic theory for this case. The correct result will be presented in two different but equivalent formulations and will be shown to lead back to well-known results from the Sommerfeld theory for the simple case with the dyes sandwiched between only one superstrate and one substrate medium.

\section{THE CPS FORMULATION}

To be clear and self-contained, let us first recapitulate the main results from the CPS article. ${ }^{4}$ For harmonic currents and fields, the dyadic Green formulation of Ref. 4 has the standard Green's function solution (in SI units):

$$
\mathbf{E}(\mathbf{R})=i \omega \mu \int \mathbf{G}\left(\mathbf{R}, \mathbf{R}^{\prime}\right) \cdot \mathbf{J}\left(\mathbf{R}^{\prime}\right) d V\left(\mathbf{R}^{\prime}\right),
$$

where $\mu$ is the magnetic permeability. For simplicity, we will consider in this paper only the case with one superstrate and one substrate confining the source in the gap as depicted in Fig. 1. More details on the case with a large number of layers and generalization to the case with gradient index media will be presented in a forthcoming paper. ${ }^{8}$

Let $\mathbf{G}_{0}$ denote the Green dyadic for the source field and $\mathbf{G}_{i}(i=1,2,3)$ denote those for the scattered fields in the three media. Thus according to Ref. 4 , one obtains ${ }^{9}$

$$
\begin{aligned}
\mathbf{G}_{0}\left(\mathbf{R}, \mathbf{R}^{\prime}\right)= & \frac{-1}{k_{1}^{2}} \hat{z} \hat{z} \delta\left(\mathbf{R}-\mathbf{R}^{\prime}\right)+\frac{i}{4 \pi} \int_{0}^{+\infty} d \lambda \sum_{n=0}^{+\infty} \frac{2-\delta_{n}}{\lambda h_{1}(\lambda)} \sum_{j=0}^{1}\left[\begin{array}{l}
\mathbf{M}_{j n \lambda}\left(+h_{1}\right) \mathbf{M}_{j n \lambda}^{\prime}\left(-h_{1}\right)+\mathbf{N}_{j n \lambda}\left(+h_{1}\right) \mathbf{N}_{j n \lambda}^{\prime}\left(-h_{1}\right) \\
\mathbf{M}_{j n \lambda}\left(-h_{1}\right) \mathbf{M}_{j n \lambda}^{\prime}\left(+h_{1}\right)+\mathbf{N}_{j n \lambda}\left(-h_{1}\right) \mathbf{N}_{j n \lambda}^{\prime}\left(+h_{1}\right)
\end{array}\right] \begin{array}{l}
z \geqslant z^{\prime} \\
z \leqslant z^{\prime}
\end{array}, \\
\mathbf{G}_{1}\left(\mathbf{R}, \mathbf{R}^{\prime}\right)= & \frac{i}{4 \pi} \int_{0}^{+\infty} d \lambda \sum_{n=0}^{+\infty} \frac{2-\delta_{n}}{\lambda h_{1}(\lambda)} \sum_{j=0}^{1}\left\{\left[c_{1} \mathbf{M}_{j n \lambda}\left(-h_{1}\right)+c_{1}^{\prime} \mathbf{M}_{j n \lambda}\left(h_{1}\right)\right] \mathbf{M}_{j n \lambda}^{\prime}\left(h_{1}\right)\right. \\
& \left.+\left[f_{1} \mathbf{N}_{j n \lambda}\left(-h_{1}\right)+f_{1}^{\prime} \mathbf{N}_{j n \lambda}\left(h_{1}\right)\right] \mathbf{N}_{j n \lambda}^{\prime}\left(h_{1}\right)\right\},
\end{aligned}
$$




$$
\begin{aligned}
\mathbf{G}_{2}\left(\mathbf{R}, \mathbf{R}^{\prime}\right)= & \frac{i}{4 \pi} \int_{0}^{+\infty} d \lambda \sum_{n=0}^{+\infty} \frac{2-\delta_{n}}{\lambda h_{1}(\lambda)} \\
& \times \sum_{j=0}^{1}\left[c_{2} \mathbf{M}_{j n \lambda}\left(h_{2}\right) \mathbf{M}_{j n \lambda}^{\prime}\left(h_{1}\right)\right. \\
& \left.+f_{2} \mathbf{N}_{j n \lambda}\left(h_{2}\right) \mathbf{N}_{j n \lambda}^{\prime}\left(h_{1}\right)\right], \\
\mathbf{G}_{3}\left(\mathbf{R}, \mathbf{R}^{\prime}\right)= & \frac{i}{4 \pi} \int_{0}^{+\infty} d \lambda \sum_{n=0}^{+\infty} \frac{2-\delta_{n}}{\lambda h_{1}(\lambda)} \\
& \times \sum_{j=0}^{1}\left[c_{3} \mathbf{M}_{j n \lambda}\left(-h_{3}\right) \mathbf{M}_{j n \lambda}^{\prime}\left(h_{1}\right)\right. \\
& \left.+f_{3} \mathbf{N}_{j n \lambda}\left(-h_{3}\right) \mathbf{N}_{j n \lambda}^{\prime}\left(h_{1}\right)\right],
\end{aligned}
$$

where $\mathbf{M}$ and $\mathbf{N}$ are given in cylindrical coordinates by:

$$
\begin{aligned}
\mathbf{M}_{j n \lambda}(h)= & e^{i h z}\left[\frac{n J_{n}(\lambda r)}{r} \sin \left(\frac{j \pi}{2}-n \varphi\right) \hat{r}-\frac{\partial J_{n}(\lambda r)}{\partial r}\right. \\
& \left.\times \cos \left(\frac{j \pi}{2}-n \varphi\right) \hat{\varphi}\right], \\
\mathbf{N}_{j n \lambda}(h)= & \frac{e^{i h z}}{k}\left[i h \frac{\partial J_{n}(\lambda r)}{\partial r} \cos \left(\frac{j \pi}{2}-n \varphi\right) \hat{r}+i n h \frac{J_{n}(\lambda r)}{r}\right. \\
& \left.\times \sin \left(\frac{j \pi}{2}-n \varphi\right) \hat{\varphi}+\lambda^{2} J_{n}(\lambda r) \cos \left(\frac{j \pi}{2}-n \varphi\right) \hat{z}\right],
\end{aligned}
$$

with $J_{n}$ the Bessel function of the first kind and $h_{i}(\lambda)$ $=\sqrt{k_{i}^{2}-\lambda^{2}}$, where the square root is taken to have positive real part.

According to Ref. 4, requirement of continuity of transverse field components at interfaces $z=0$ and $z=z_{0}$ leads to the following systems where $e_{j} \equiv e^{i h_{j} z_{0}}$ :

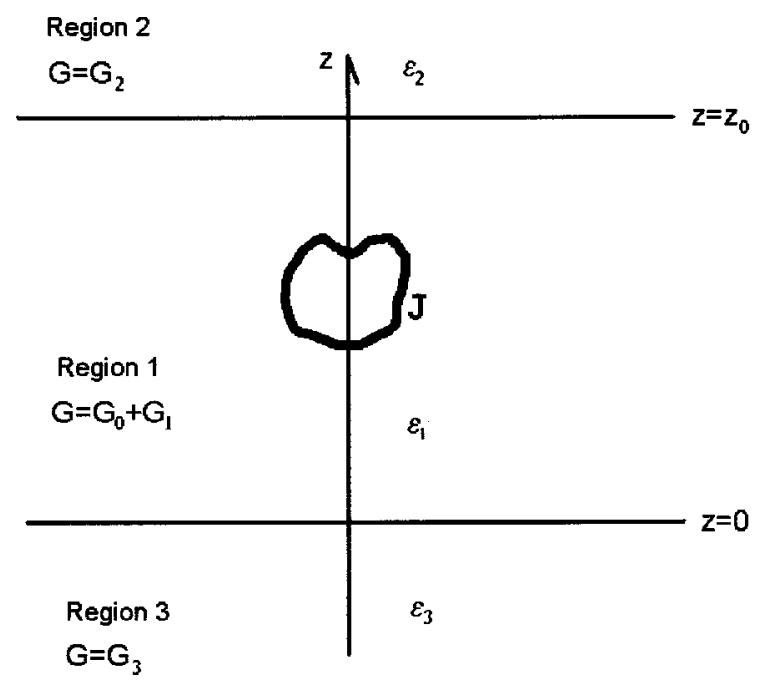

FIG. 1. Geometry of the problem.

$$
\left[\begin{array}{cccc}
1 & 1 & 0 & -1 \\
-h_{1} & h_{1} & 0 & h_{3} \\
\frac{1}{e_{1}} & e_{1} & -e_{2} & 0 \\
-\frac{h_{1}}{e_{1}} & h_{1} e_{1} & -h_{2} e_{2} & 0
\end{array}\right]\left[\begin{array}{c}
c_{1} \\
c_{1}^{\prime} \\
c_{2} \\
c_{3}
\end{array}\right]=\left[\begin{array}{c}
-1 \\
h_{1} \\
-e_{1} \\
-h_{1} e_{1}
\end{array}\right]
$$

or in matrix form $\mathbf{A c}=\mathbf{r}_{c}$, and

$$
\begin{gathered}
{\left[\begin{array}{cccc}
-h_{1} / k_{1} & h_{1} / k_{1} & 0 & h_{3} / k_{3} \\
k_{1} & k_{1} & 0 & -k_{3} \\
\frac{-h_{1}}{k_{1} e_{1}} & h_{1} e_{1} / k_{1} & -h_{2} e_{2} / k_{2} & 0 \\
\frac{k_{1}}{e_{1}} & k_{1} e_{1} & -k_{2} e_{2} & 0
\end{array}\right]\left[\begin{array}{l}
f_{1} \\
f_{1}^{\prime} \\
f_{2} \\
f_{3}
\end{array}\right]} \\
=\left[\begin{array}{c}
h_{1} / k_{1} \\
-k_{1} \\
-e_{1} h_{1} / k_{1} \\
-e_{1} k_{1}
\end{array}\right],
\end{gathered}
$$

or $\mathbf{B f}=\mathbf{r}_{f}$. Solving Eqs. (8) and (9) yields the following:

$$
\left(\begin{array}{c}
c_{1} \\
c_{1}^{\prime} \\
f_{1} \\
f_{1}^{\prime}
\end{array}\right)=\left(\begin{array}{c}
e_{1}^{2} R_{12}^{\perp} \frac{1+R_{13}^{\perp}}{1-e_{1}^{2} R_{12}^{\perp} R_{13}^{\perp}} \\
R_{13}^{\perp} \frac{1+e_{1}^{2} R_{12}^{\perp}}{1-e_{1}^{2} R_{12}^{\perp} R_{13}^{\perp}} \\
-R_{12}^{\|} \frac{e_{1}^{2}\left(1-R_{13}^{\|}\right)}{1-e_{1}^{2} R_{12}^{\|} R_{13}^{\|}} \\
-R_{13}^{\|} \frac{\left(1-e_{1}^{2} R_{12}^{\|}\right)}{1-e_{1}^{2} R_{12}^{\|} R_{13}^{\|}}
\end{array}\right),
$$

where

$$
R_{i j}^{\|} \equiv \frac{\epsilon_{i} h_{j}-\epsilon_{j} h_{i}}{\epsilon_{i} h_{j}+\epsilon_{j} h_{i}}
$$

and

$$
R_{i j}^{\perp} \equiv \frac{h_{i}-h_{j}}{h_{i}+h_{j}} .
$$

Note that sign errors in Eq. (3.34) of Ref. 4 are corrected in Eq. (10).

We have confirmed that the above results are in error by performing a numerical calculation of special cases. For example, the scattered electric field was calculated at the site of a vertical dipole located at the center of region 1 , with values for the dielectric constants $\epsilon_{1}, \epsilon_{2}$, and $\epsilon_{3}$ set arbitrarily. Next the values of $\epsilon_{2}$ and $\epsilon_{3}$ were interchanged. Results in the two cases differed, indicating that the analytical results in Eq. (10) are in error.

\section{THE CORRECT SOLUTION}

We shall present here two different approaches to the correct Green dyadic solution to the above problem. 


\section{A. Solution by expanding the solution space}

In reviewing the problem, we found that Eqs. (8) and (9) do not satisfy the boundary conditions at the interfaces and that no solution could be found once the constraints of forms (3) (4), and (5) were imposed. A necessary remedy is enlargement of the solution space to the point where the bound- ary conditions can be satisfied. For instance, the expression for $\mathbf{G}_{1}$ contains dyadic products $\mathbf{M}_{j n \lambda}\left(-h_{1}\right) \mathbf{M}_{j n \lambda}^{\prime}\left(+h_{1}\right)$ and $\mathbf{M}_{j n \lambda}\left(+h_{1}\right) \mathbf{M}_{j n \lambda}^{\prime}\left(+h_{1}\right)$ but not $\mathbf{M}_{j n \lambda}\left(-h_{1}\right) \mathbf{M}_{j n \lambda}^{\prime}\left(-h_{1}\right)$ and $\mathbf{M}_{j n \lambda}\left(+h_{1}\right) \mathbf{M}_{j n \lambda}^{\prime}\left(-h_{1}\right)$ which are equally valid. It turns out that the correct solution from this approach has already been worked out in the electrical engineering literature. ${ }^{10}$ The general solutions for the scattering fields are given by

$$
\begin{aligned}
\mathbf{G}_{1}\left(\mathbf{R}, \mathbf{R}^{\prime}\right) \equiv & \frac{i}{4 \pi} \int_{0}^{+\infty} d \lambda \sum_{n=0}^{+\infty} \frac{2-\delta_{n}}{\lambda h_{1}(\lambda)} \sum_{j=0}^{1}\left\{\left[c_{1} \mathbf{M}_{j n \lambda}\left(-h_{1}\right)+c_{1}^{\prime} \mathbf{M}_{j n \lambda}\left(h_{1}\right)\right] \mathbf{M}_{j n \lambda}^{\prime}\left(h_{1}\right)\right. \\
& +\left[a_{1} \mathbf{M}_{j n \lambda}\left(-h_{1}\right)+a_{1}^{\prime} \mathbf{M}_{j n \lambda}\left(h_{1}\right)\right] \mathbf{M}_{j n \lambda}^{\prime}\left(-h_{1}\right)+\left[f_{1} \mathbf{N}_{j n \lambda}\left(-h_{1}\right)+f_{1}^{\prime} \mathbf{N}_{j n \lambda}\left(h_{1}\right)\right] \mathbf{N}_{j n \lambda}^{\prime}\left(h_{1}\right) \\
& \left.+\left[b_{1} \mathbf{N}_{j n \lambda}\left(-h_{1}\right)+b_{1}^{\prime} \mathbf{N}_{j n \lambda}\left(h_{1}\right)\right] \mathbf{N}_{j n \lambda}^{\prime}\left(-h_{1}\right)\right\}, \\
\mathbf{G}_{2}\left(\mathbf{R}, \mathbf{R}^{\prime}\right) \equiv & \frac{i}{4 \pi} \int_{0}^{+\infty} d \lambda \sum_{n=0}^{+\infty} \frac{2-\delta_{n}}{\lambda h_{1}(\lambda)} \sum_{j=0}^{1}\left[c_{2} \mathbf{M}_{j n \lambda}\left(h_{2}\right) \mathbf{M}_{j n \lambda}^{\prime}\left(h_{1}\right)+f_{2} \mathbf{N}_{j n \lambda}\left(h_{2}\right) \mathbf{N}_{j n \lambda}^{\prime}\left(h_{1}\right)+a_{2} \mathbf{M}_{j n \lambda}\left(h_{2}\right) \mathbf{M}_{j n \lambda}^{\prime}\left(-h_{1}\right)\right. \\
& \left.+b_{2} \mathbf{N}_{j n \lambda}\left(h_{2}\right) \mathbf{N}_{j n \lambda}^{\prime}\left(-h_{1}\right)\right], \\
\mathbf{G}_{3}\left(\mathbf{R}, \mathbf{R}^{\prime}\right) \equiv & \frac{i}{4 \pi} \int_{0}^{+\infty} d \lambda \sum_{n=0}^{+\infty} \frac{2-\delta_{n}}{\lambda h_{1}(\lambda)} \sum_{j=0}^{1}\left[c_{3} \mathbf{M}_{j n \lambda}\left(-h_{3}\right) \mathbf{M}_{j n \lambda}^{\prime}\left(h_{1}\right)+f_{3} \mathbf{N}_{j n \lambda}\left(-h_{3}\right) \mathbf{N}_{j n \lambda}^{\prime}\left(h_{1}\right)\right. \\
& \left.+a_{3} \mathbf{M}_{j n \lambda}\left(-h_{3}\right) \mathbf{M}_{j n \lambda}^{\prime}\left(-h_{1}\right)+b_{3} \mathbf{N}_{j n \lambda}\left(-h_{3}\right) \mathbf{N}_{j n \lambda}^{\prime}\left(-h_{1}\right)\right] .
\end{aligned}
$$

By imposing the appropriate boundary conditions, the expansion coefficients can finally be obtained as ${ }^{10}$

$$
\begin{gathered}
\mathbf{c}_{s}=\frac{1}{1-e_{1}^{2} R_{12}^{\perp} R_{13}^{\perp}}\left[\begin{array}{c}
e_{1}^{2} R_{12}^{\perp} R_{13}^{\perp} \\
R_{13}^{\perp} \\
\frac{e_{1}}{e_{2}}\left(1+R_{12}^{\perp}\right) R_{13}^{\perp} \\
\left(1+R_{13}^{\perp}\right)
\end{array}\right], \\
\mathbf{a}_{s}=\frac{1}{1-e_{1}^{2} R_{12}^{\perp} R_{13}^{\perp}}\left[\begin{array}{c}
e_{1}^{2} R_{12}^{\perp} \\
e_{1}^{2} R_{12}^{\perp} R_{13}^{\perp} \\
\frac{e_{1}}{e_{2}}\left(1+R_{12}^{\perp}\right) \\
e_{1}^{2} R_{12}^{\perp}\left(1+R_{13}^{\perp}\right)
\end{array}\right], \\
\mathbf{f}_{s}=\frac{1}{1-e_{1}^{2} R_{12}^{\|} R_{13}^{\|}}\left[\begin{array}{c}
e_{1}^{2} R_{12}^{\|} R_{13}^{\|} \\
-R_{13}^{\|} \\
\frac{-k_{1} e_{1}}{k_{2} e_{2}}\left(1-R_{12}^{\|}\right) R_{13}^{\|} \\
\frac{k_{1}}{k_{3}}\left(1-R_{13}^{\|}\right) \\
-e_{1}^{2} R_{12}^{\|} \\
e_{1}^{2} R_{12}^{\|} R_{13}^{\|} \\
\frac{k_{1} e_{1}}{k_{2} e_{2}}\left(1-R_{12}^{\|}\right) \\
\frac{-k_{1}}{k_{3}} e_{1}^{2} R_{12}^{\|}\left(1-R_{13}^{\|}\right)
\end{array}\right], \\
\mathbf{b}_{s}=\frac{1}{1-e_{1}^{2} R_{12}^{\|} R_{13}^{\|}}\left[\begin{array}{c} 
\\
{\left[\begin{array}{c}
\| \\
n_{1}
\end{array}\right.}
\end{array}\right],
\end{gathered}
$$

where

$$
\mathbf{c}_{s}=\left[\begin{array}{l}
c_{1} \\
c_{1}^{\prime} \\
c_{2} \\
c_{3}
\end{array}\right], \quad \mathbf{a}_{s}=\left[\begin{array}{l}
a_{1} \\
a_{1}^{\prime} \\
a_{2} \\
a_{3}
\end{array}\right], \mathbf{f}_{s}=\left[\begin{array}{l}
f_{1} \\
f_{1}^{\prime} \\
f_{2} \\
f_{3}
\end{array}\right],
$$

and

$$
\mathbf{b}_{s}=\left[\begin{array}{l}
b_{1} \\
b_{1}^{\prime} \\
b_{2} \\
b_{3}
\end{array}\right]
$$

Using the above solution, we can write out the Green's functions as

$$
\begin{aligned}
\mathbf{G}_{1}\left(\mathbf{R}, \mathbf{R}^{\prime}\right) \equiv & \frac{i}{4 \pi} \int_{0}^{+\infty} d \lambda \sum_{n=0}^{+\infty} \frac{2-\delta_{n}}{\lambda h_{1}(\lambda)} \sum_{j=0}^{1}\left(\frac{1}{1-e_{1}^{2} R_{12}^{\perp} R_{13}^{\perp}}\right. \\
& \times\left[e_{1}^{2} R_{12}^{\perp} \mathbf{M}^{-}\left(R_{13}^{\perp} \mathbf{M}^{+}+\mathbf{M}^{-}\right)+R_{13}^{\perp} \mathbf{M}^{+}\left(\mathbf{M}^{+}\right.\right. \\
& \left.\left.+e_{1}^{2} R_{12}^{\perp} \mathbf{M}^{-}\right)\right]+\frac{1}{1-e_{1}^{2} R_{12}^{\|} R_{13}^{\|}} \\
& \times\left[e_{1}^{2} R_{12}^{\|} \mathbf{N}^{-}\left(R_{13}^{\|} \mathbf{N}^{+}-\mathbf{N}^{-}\right)\right. \\
& \left.\left.+R_{13}^{\|} \mathbf{N}^{+}\left(e_{1}^{2} R_{12}^{\|} \mathbf{N}^{-}-\mathbf{N}^{+}\right)\right]\right),
\end{aligned}
$$




$$
\begin{aligned}
\mathbf{G}_{2}\left(\mathbf{R}, \mathbf{R}^{\prime}\right) \equiv & \frac{i}{4 \pi} \int_{0}^{+\infty} d \lambda \sum_{n=0}^{+\infty} \frac{2-\delta_{n}}{\lambda h_{1}(\lambda)} \sum_{j=0}^{1}\left[\frac{1}{1-e_{1}^{2} R_{12}^{\perp} R_{13}^{\perp}}\left(\frac{e_{1}}{e_{2}}\left(1+R_{12}^{\perp}\right) \mathbf{M}\left(h_{2}\right)\left(\mathbf{M}^{-}+R_{13}^{\perp} \mathbf{M}^{+}\right)\right)\right. \\
& \left.+\frac{1}{1-e_{1}^{2} R_{12}^{\|} R_{13}^{\|}}\left(\frac{k_{1} e_{1}}{k_{2} e_{2}}\left(1-R_{12}^{\|}\right) \mathbf{N}\left(h_{2}\right)\left(\mathbf{N}^{-}-R_{13}^{\|} \mathbf{N}^{+}\right)\right)\right], \\
\mathbf{G}_{3}\left(\mathbf{R}, \mathbf{R}^{\prime}\right) \equiv & \frac{i}{4 \pi} \int_{0}^{+\infty} d \lambda \sum_{n=0}^{+\infty} \frac{2-\delta_{n}}{\lambda h_{1}(\lambda)} \sum_{j=0}^{1}\left[\frac{1}{1-e_{1}^{2} R_{12}^{\perp} R_{13}^{\perp}}\left[\left(1+R_{13}^{\perp}\right) \mathbf{M}\left(-h_{3}\right)\left(\mathbf{M}^{+}+e_{1}^{2} R_{12}^{\perp} \mathbf{M}^{-}\right)\right]\right. \\
& \left.+\frac{1}{1-e_{1}^{2} R_{12}^{\|} R_{13}^{\|}}\left(\frac{k_{1}}{k_{3}}\left(1-R_{13}^{\|}\right) \mathbf{N}\left(-h_{3}\right)\left(\mathbf{N}^{+}-e_{1}^{2} R_{12}^{\|} \mathbf{N}^{-}\right)\right)\right],
\end{aligned}
$$

where $\mathbf{M}^{ \pm}=\mathbf{M}^{\prime}\left( \pm h_{1}\right)$ and $\mathbf{N}^{ \pm}=\mathbf{N}^{\prime}\left( \pm h_{1}\right)$. We have checked that the solution given by Eqs. (15) $-(17)$ does satisfy the numerical test described above.

\section{B. Solution by reassociation}

An alternative approach, which might be called "reassociation", is to introduce explicitly the source $\mathbf{J}$ into the dyadic expansion. We replaced typical products such as $\left(\mathbf{M} \mathbf{M}^{\prime}\right) \mathbf{J}$ with the equivalent product $\mathbf{M}\left(\mathbf{M}^{\prime} \cdot \mathbf{J}\right)$ reducing the product on the right to a complex scalar. An additional small step then leads to the realization that $c\left(\mathbf{M M}^{\prime}\right) \mathbf{J}$ can be replaced by $c \mathbf{M}$, where $\mathbf{M}^{\prime} \cdot \mathbf{J}$ scalar has been absorbed into the $c$. We will see at the end that $\mathbf{J}$ can be factored from both sides of the resulting equations leading to expressions for $\mathbf{G}_{i}$ independent of the source, as they must be. Following the logic given above, we have:

$$
\begin{aligned}
& \mathbf{G}_{1}\left(\mathbf{R}, \mathbf{R}^{\prime}\right) \cdot \mathbf{J} \equiv \frac{i}{4 \pi} \int_{0}^{+\infty} d \lambda \sum_{n=0}^{+\infty} \frac{2-\delta_{n}}{\lambda h_{1}(\lambda)} \sum_{j=0}^{1}\left[c_{1} \mathbf{M}_{j n \lambda}\left(-h_{1}\right)+c_{1}^{\prime} \mathbf{M}_{j n \lambda}\left(h_{1}\right)+f_{1} \mathbf{N}_{j n \lambda}\left(-h_{1}\right)+f_{1}^{\prime} \mathbf{N}_{j n \lambda}\left(h_{1}\right)\right] \\
& \mathbf{G}_{2}\left(\mathbf{R}, \mathbf{R}^{\prime}\right) \cdot \mathbf{J} \equiv \frac{i}{4 \pi} \int_{0}^{+\infty} d \lambda \sum_{n=0}^{+\infty} \frac{2-\delta_{n}}{\lambda h_{1}(\lambda)} \sum_{j=0}^{1}\left[c_{2} \mathbf{M}_{j n \lambda}\left(h_{2}\right)+f_{2} \mathbf{N}_{j n \lambda}\left(h_{2}\right)\right] \\
& \mathbf{G}_{3}\left(\mathbf{R}, \mathbf{R}^{\prime}\right) \cdot \mathbf{J} \equiv \frac{i}{4 \pi} \int_{0}^{+\infty} d \lambda \sum_{n=0}^{+\infty} \frac{2-\delta_{n}}{\lambda h_{1}(\lambda)} \sum_{j=0}^{1}\left[c_{3} \mathbf{M}_{j n \lambda}\left(-h_{3}\right)+f_{3} \mathbf{N}_{j n \lambda}\left(-h_{3}\right)\right]
\end{aligned}
$$

where the $c$ and $f$ coefficients are functions of current density $\mathbf{J}$ as well as position, $j, n$ and $\lambda$. This approach allows us to work directly with electric and magnetic field values in applying the boundary conditions at the interfaces. The resulting eight equations decouple into two matrix systems:

$$
\begin{aligned}
& \mathbf{A c}=\left\{\left[\begin{array}{llll}
-\mathbf{M}^{\prime}\left(h_{1}\right) & h_{1} \mathbf{M}^{\prime}\left(h_{1}\right) & -e_{1} \mathbf{M}^{\prime}\left(-h_{1}\right) & -h_{1} e_{1} \mathbf{M}^{\prime}\left(-h_{1}\right)
\end{array}\right] \cdot \mathbf{J}\right\}^{t}, \\
& \left.\mathbf{B f}=\left[\begin{array}{llll}
\frac{h_{1}}{k_{1}} \mathbf{N}^{\prime}\left(h_{1}\right) & -k_{1} \mathbf{N}^{\prime}\left(h_{1}\right) & -\frac{h_{1} e_{1}}{k_{1}} \mathbf{N}^{\prime}\left(-h_{1}\right) & -k_{1} e_{1} \mathbf{N}^{\prime}\left(-h_{1}\right)
\end{array}\right) \cdot \mathbf{J}\right]^{t},
\end{aligned}
$$

where $\mathbf{A}$ and $\mathbf{B}$ are the same matrices defined above in Eqs. (8) and (9). The matrix solution of Eqs. (21) and (22) leads to:

$$
\begin{aligned}
& {\left[\begin{array}{l}
c_{1} \\
c_{1}^{\prime} \\
c_{2} \\
c_{3}
\end{array}\right]=\frac{1}{1-e_{1}^{2} R_{12}^{\perp} R_{13}^{\perp}}\left[\begin{array}{c}
e_{1}^{2} R_{12}^{\perp}\left(R_{13}^{\perp} \mathbf{M}^{+}+\mathbf{M}^{-}\right) \\
R_{13}^{\perp}\left(\mathbf{M}^{+}+e_{1}^{2} R_{12}^{\perp} \mathbf{M}^{-}\right) \\
\frac{e_{1}}{e_{2}}\left(1+R_{12}^{\perp}\right)\left(\mathbf{M}^{-}+R_{13}^{\perp} \mathbf{M}^{+}\right) \\
\left(1+R_{13}^{\perp}\right)\left(\mathbf{M}^{+}+e_{1}^{2} R_{12}^{\perp} \mathbf{M}^{-}\right)
\end{array}\right] \cdot \mathbf{J}} \\
& {\left[\begin{array}{l}
f_{1} \\
f_{1}^{\prime} \\
f_{2} \\
f_{3}
\end{array}\right]=\frac{1}{1-e_{1}^{2} R_{12}^{\|} R_{13}^{\|}}\left[\begin{array}{c}
e_{1}^{2} R_{12}^{\|}\left(R_{13}^{\|} \mathbf{N}^{+}-\mathbf{N}^{-}\right) \\
\frac{k_{13} e_{1}}{k_{2} e_{2}}\left(1-e_{1}^{2} R_{12}^{\|} \mathbf{N}^{-}-\mathbf{N}^{+}\right) \\
\frac{k_{1}}{k_{3}}\left(1-R_{13}^{\|}\right)\left(\mathbf{N}^{-}-R_{13}^{\|} \mathbf{N}^{+}\right)
\end{array}\right] \cdot \mathbf{J} .}
\end{aligned}
$$

We can now insert Eqs. (23) and (24) into Eqs. (18)-(20). Since an arbitrary $\mathbf{J}$ then appears on both sides of the result, we can factor out $\mathbf{J}$, yielding results in complete agreement with Eqs. (15)-(17). More details on this approach and the equivalence between the two methods will be provided in a forthcoming paper. ${ }^{8}$

\section{CALCULATION OF DECAY RATES}

According to the classical phenomenological approach of CPS, the normalized decay rate of the admolecule can be obtained in terms of the imaginary part $(\mathfrak{I})$ of the reflected field at the dipole site as:

$$
\hat{b} \equiv \frac{b}{b_{0}}=1+\frac{6 \pi \epsilon_{0} q n_{1}^{2}}{p_{0} k_{1}^{3}} \mathfrak{I}\left(\mathbf{E}_{0}\right),
$$


where $q$ is the intrinsic quantum yield and $k_{1}=n_{1} \omega / c$, with $n_{1}$ the real refractive index of the medium containing the dipole. We show below that the dyadics given in Eqs. (15)(17) can indeed lead back to the correct results for $\hat{b}$ for a molecule confined as in the geometry of Fig. 1.

We first consider the problem of a vertically oriented dipole with moment $p_{0} \hat{z} e^{-i \omega t}$ at the source position $d \hat{z}$ between two interfaces at $z=0$ and $z=s+d=z_{0}$. The current will be given by

$$
\mathbf{J}=-i \omega p_{0} \hat{z} e^{-i \omega t} \delta\left(\mathbf{R}^{\prime}-d \hat{z}\right) .
$$

Inserting this $\mathbf{J}$ into Eqs. (1) and using Eq. (15) yields

$$
\begin{aligned}
\mathbf{E}_{z}^{\perp}(d \hat{z})= & \left\{\omega^{2} \mu_{0} \mu_{1} p_{0} e^{-i \omega t}\right\} \hat{z} \cdot \mathbf{G}_{1}(d \hat{z}, d \hat{z}) \hat{z} \\
= & \left\{\omega^{2} \mu_{0} \mu_{1} p_{0} e^{-i \omega t}\right\} \frac{i}{4 \pi} \int_{\lambda=0}^{+\infty} \sum_{n=0}^{+\infty} \frac{2-\delta_{n}}{\lambda h_{1}(\lambda)} \\
& \times \sum_{j=0,1} \hat{z} \cdot\left(\frac{1}{1-e_{1}^{2} R_{12}^{\|} R_{13}^{\|}}\left[e_{1}^{2} R_{12}^{\|} \mathbf{N}^{-}\left(R_{13}^{\|} \mathbf{N}^{+}-\mathbf{N}^{-}\right)+R_{13}^{\|} \mathbf{N}^{+}\left(e_{1}^{2} R_{12}^{\|} \mathbf{N}^{-}-\mathbf{N}^{+}\right)\right]\right) \hat{z} d \lambda \\
= & \left\{\omega^{2} \mu_{0} \mu_{1} p_{0} e^{-i \omega t}\right\} \frac{i}{4 \pi} \int_{\lambda=0}^{+\infty} \frac{1}{\lambda h_{1}(\lambda)}\left\{\frac { 1 } { 1 - e _ { 1 } ^ { 2 } R _ { 1 2 } ^ { \| } R _ { 1 3 } ^ { \| } } \left[e_{1}^{2} R_{12}^{\|} \frac{\lambda^{2}}{e_{d} k_{1}}\left(R_{13}^{\|} \frac{e_{d} \lambda^{2}}{k_{1}}-\frac{\lambda^{2}}{e_{d} k_{1}}\right)\right.\right. \\
& \left.\left.+R_{13}^{\|} \frac{e_{d} \lambda^{2}}{k_{1}}\left(e_{1}^{2} R_{12}^{\|} \frac{\lambda^{2}}{e_{d} k_{1}}-\frac{e_{d} \lambda^{2}}{k_{1}}\right)\right]\right\} d \lambda \\
= & \frac{i \omega^{2} \mu_{0} \mu_{1} p_{0} e^{-i \omega t}}{4 \pi k_{1}^{2}} \int_{\lambda=0}^{+\infty} \frac{\lambda^{3}}{h_{1}(\lambda)} \frac{\left(2 e_{1}^{2} R_{12}^{\|} R_{13}^{\|}-e_{s}^{2} R_{12}^{\|}-e_{d}^{2} R_{13}^{\|}\right)}{\left(1-e_{1}^{2} R_{12}^{\|} R_{13}^{\|}\right)} d \lambda \\
= & \frac{p_{0} e^{-i \omega t}}{4 \pi \epsilon_{0} \epsilon_{1}} \int_{\lambda=0}^{+\infty} \frac{\lambda^{3}}{\left[-i h_{1}(\lambda)\right]}\left(\frac{\left(1-R_{13}^{\|} e_{d}^{2}\right)\left(1-R_{12}^{\|} e_{s}^{2}\right)}{\left(1-e_{1}^{2} R_{12}^{\|} R_{13}^{\|}\right)}-1\right) d \lambda,
\end{aligned}
$$

where $e_{d}=e^{i d h_{1}(\lambda)}$ and $e_{s}=e^{i s h_{1}(\lambda)}$ with $s+d=z_{0}$. We have also employed the identity:

$$
\frac{2 x y-x-y}{1-x y}=\frac{(1-x)(1-y)}{1-x y}-1 \text {. }
$$

Inserting Eq. (27) into Eq. (25), we obtain

$$
\begin{aligned}
\hat{b}_{\perp}= & 1+\frac{6 \pi \epsilon_{0} q n_{1}^{2}}{p_{0} k_{1}^{3}} \Im\left[\frac{p_{0}}{4 \pi \epsilon_{0} \epsilon_{1}} \int_{\lambda=0}^{+\infty} \frac{\lambda^{3}}{\left[-i h_{1}(\lambda)\right]}\right. \\
& \left.\times\left(\frac{\left(1-R_{13}^{\|} e_{d}^{2}\right)\left(1-R_{12}^{\|} e_{s}^{2}\right)}{\left(1-e_{1}^{2} R_{12}^{\|} R_{13}^{\|}\right)}-1\right) d \lambda\right] \\
= & 1-q+\frac{3 q}{2 k_{1}^{3}} \\
& \times \Im\left[i \int_{\lambda=0}^{+\infty} \frac{\lambda^{3}}{h_{1}(\lambda)}\left(\frac{\left(1-R_{13}^{\|} e_{d}^{2}\right)\left(1-R_{12}^{\|} e_{s}^{2}\right)}{\left(1-e_{1}^{2} R_{12}^{\|} R_{13}^{\|}\right)}\right) d \lambda\right],
\end{aligned}
$$

where we have used

$$
\int_{\lambda=0}^{k} \frac{\lambda^{3} d \lambda}{\sqrt{k^{2}-\lambda^{2}}}=\frac{2}{3} k^{3} .
$$

The result in Eq. (28) is equivalent to Eq. (2.47) of Ref. 4 using the transformation: $u=\lambda / k_{1}$. In the case of a trivial interface between regions 1 and 2, that is, $\epsilon_{1}=\epsilon_{2}$ implying $R_{12}^{\|}=0$, from Eq. (28) we have

$$
\hat{b}_{\perp}=1-\frac{3 q}{2 k_{1}^{3}} \Im\left(i \int_{\lambda=0}^{+\infty} R_{13}^{\|} e_{d}^{2} \frac{\lambda^{3} d \lambda}{h_{1}(\lambda)}\right),
$$

which is identical to Eq. (2.17) of Ref. 4.

We next consider the problem of a horizontally oriented dipole with moment $p_{0} \hat{x} e^{-i \omega t}$ at the source position $d \hat{z}$ between the same interfaces. The current density is then

$$
\mathbf{J}=-i \omega p_{0} \hat{x} e^{-i \omega t} \delta\left(\mathbf{R}^{\prime}-d \hat{z}\right) .
$$

Again, inserting $\mathbf{J}$ into Eq. (1) and integrating yields

$$
E_{x}^{\|}(d \hat{z})=\left\{\omega^{2} \mu_{0} \mu_{1} p_{0} e^{-i \omega t}\right\} \hat{x} \cdot \mathbf{G}_{1}(d \hat{z}, d \hat{z}) \hat{x} .
$$

To proceed further, we note that

$$
\hat{x} \cdot \mathbf{M}^{ \pm}(d \hat{z})=\left\{\begin{array}{l}
\frac{\lambda}{2} e^{ \pm i h_{1} d} \quad \text { if } n=1 \quad \text { and } j=1 \\
0 \quad \text { otherwise }
\end{array}\right.
$$

and

$$
\hat{x} \cdot \mathbf{N}^{ \pm}(d \hat{z})=\left\{\begin{array}{l}
\frac{ \pm i \lambda h_{1}}{2 k_{1}} e^{ \pm i h_{1} d} \quad \text { if } n=1 \quad \text { and } j=0 \\
0 \quad \text { otherwise }
\end{array}\right.
$$

Insertion of the above dot products into Eq. (15) yields 


$$
\begin{aligned}
\hat{x} \cdot \mathbf{G}_{1}(d \hat{z}, d \hat{z}) \cdot \hat{x}= & \frac{i}{8 \pi} \int_{\lambda=0}^{+\infty}\left(\frac{1}{1-e_{1}^{2} R_{12}^{\perp} R_{13}^{\perp}}\left(2 e_{1}^{2} R_{12}^{\perp} R_{13}^{\perp}+e_{s}^{2} R_{12}^{\perp}+e_{d}^{2} R_{13}^{\perp}\right)\right. \\
& \left.+\frac{h_{1}^{2}(\lambda)}{k_{1}^{2}\left(1-e_{1}^{2} R_{12}^{\|} R_{13}^{\|}\right)}\left(2 e_{1}^{2} R_{12}^{\|} R_{13}^{\|}+e_{s}^{2} R_{12}^{\|}+e_{d}^{2} R_{13}^{\|}\right)\right) \frac{\lambda d \lambda}{h_{1}(\lambda)} \\
= & \frac{i}{8 \pi k_{1}^{2}} \int_{\lambda=0}^{+\infty}\left[k_{1}^{2}\left(\frac{\left(e_{s}^{2} R_{12}^{\perp}+1\right)\left(e_{d}^{2} R_{13}^{\perp}+1\right)}{1-e_{1}^{2} R_{12}^{\perp} R_{13}^{\perp}}-1\right)+h_{1}^{2}(\lambda)\left(\frac{\left(e_{s}^{2} R_{12}^{\|}+1\right)\left(e_{d}^{2} R_{13}^{\|}+1\right)}{1-e_{1}^{2} R_{12}^{\|} R_{13}^{\|}}-1\right)\right] \frac{\lambda d \lambda}{h_{1}(\lambda)},
\end{aligned}
$$

where we have again used the identity:

$$
\frac{2 x y+x+y}{1-x y}=\frac{(x+1)(y+1)}{1-x y}-1 .
$$

Using $k_{1}^{2}=\omega^{2} \epsilon_{0} \epsilon_{1} \mu_{0} \mu_{1}$, we finally have

$$
E_{x}^{\|}(d \hat{z})=\frac{p_{0} i e^{-i \omega t}}{8 \pi \epsilon_{0} \epsilon_{1}} \int_{\lambda=0}^{+\infty}\left[k_{1}^{2}\left(\frac{\left(e_{s}^{2} R_{12}^{\perp}+1\right)\left(e_{d}^{2} R_{13}^{\perp}+1\right)}{1-e_{1}^{2} R_{12}^{\perp} R_{13}^{\perp}}-1\right)+h_{1}^{2}(\lambda)\left(\frac{\left(e_{s}^{2} R_{12}^{\|}+1\right)\left(e_{d}^{2} R_{13}^{\|}+1\right)}{1-e_{1}^{2} R_{12}^{\|} R_{13}^{\|}}-1\right)\right] \frac{\lambda d \lambda}{h_{1}(\lambda)} .
$$

Inserting Eq. (32) into Eq. (25), we obtain

$$
\begin{aligned}
\hat{b}_{\|} & =1+\frac{6 \pi \epsilon_{0} q n_{1}^{2}}{p_{0} k_{1}^{3}} \mathfrak{I}\left\{\frac{p_{0}}{8 \pi \epsilon_{0} \epsilon_{1}} \int_{\lambda=0}^{+\infty}\left[k_{1}^{2}\left(\frac{\left(e_{s}^{2} R_{12}^{\perp}+1\right)\left(e_{d}^{2} R_{13}^{\perp}+1\right)}{1-e_{1}^{2} R_{12}^{\perp} R_{13}^{\perp}}-1\right)+h_{1}^{2}(\lambda)\left(\frac{\left(e_{s}^{2} R_{12}^{\|}+1\right)\left(e_{d}^{2} R_{13}^{\|}+1\right)}{1-e_{1}^{2} R_{12}^{\|} R_{13}^{\|}}-1\right)\right] \frac{\lambda d \lambda}{\left[-i h_{1}(\lambda)\right]}\right\} \\
& =1-q+\frac{3 q}{4 k_{1}^{3}} \Im\left\{\int_{\lambda=0}^{+\infty}\left[k_{1}^{2}\left(\frac{\left(e_{s}^{2} R_{12}^{\perp}+1\right)\left(e_{d}^{2} R_{13}^{\perp}+1\right)}{1-e_{1}^{2} R_{12}^{\perp} R_{13}^{\perp}}\right)+h_{1}^{2}(\lambda)\left(\frac{\left(e_{s}^{2} R_{12}^{\|}+1\right)\left(e_{d}^{2} R_{13}^{\|}+1\right)}{1-e_{1}^{2} R_{12}^{\|} R_{13}^{\|}}\right)\right] \frac{\lambda d \lambda}{\left[-i h_{1}(\lambda)\right]}\right\},
\end{aligned}
$$

where we have used

$$
\int_{\lambda=0}^{k} \frac{\left(2 k^{2}-\lambda^{2}\right) \lambda d \lambda}{\sqrt{k^{2}-\lambda^{2}}}=\frac{4}{3} k^{3} .
$$

The result in Eq. (33) is equivalent to Eq. (2.48) of Ref. 4 using the transformation: $u=\lambda / k_{1}$. In the case of a trivial interface between regions 1 and 2, that is $\epsilon_{1}=\epsilon_{2}$, implying that $R_{12}^{\perp}=R_{12}^{\|}=0$, we have from Eq. (33)

$$
\hat{b}_{\|}=1+\frac{3 q}{4 k_{1}^{3}} \Im\left(i \int_{\lambda=0}^{+\infty} e_{d}^{2}\left[k_{1}^{2} R_{13}^{\perp}+h_{1}^{2}(\lambda) R_{13}^{\|}\right] \frac{\lambda d \lambda}{h_{1}(\lambda)}\right),
$$

which is identical to Eq. (2.29) of Ref. 4. Thus our Green dyadics in Eqs. (15)-(17) indeed reproduce the correct results for the decay rates obtained by the generalization of the Sommerfeld method. ${ }^{4}$

\section{CONCLUSION}

The dyadic Green's function solution to the double mirror problem in Ref. 4 was found to have a theoretical error. We have shown that the error can be corrected by extending and symmetrizing the solution form in order to satisfy boundary conditions as done in Ref. 10. Alternatively, reassociation of the dyadic product with current density leads to the same solution as can be seen by virtue of an isomorphism between formulations. ${ }^{8}$ Finally, the corrected dyadic Green's function formulation can be used directly to calculate and verify decay rates calculated in Ref. 4 from the Sommerfeld theory for the case of an oscillating dipole positioned between interfaces. With either of the two approaches, generalization is straightforward to the case with an arbitrary number of multiple interfaces for both the substrate and superstrate. ${ }^{8,10}$ In addition, the green dydadic formalism will also allow one to calculate an arbitrary source within the gap beyond that of an electric point dipole. The present formulation should be useful in these aspects.

\section{ACKNOWLEDGMENTS}

The authors are grateful to Professor Robert Silbey of MIT for very helpful correspondence. Partial support from Oregon Medical Systems is also acknowledged.

${ }^{1}$ H. Kuhn, J. Chem. Phys. 53, 101 (1970).

${ }^{2}$ K. H. Drexhage, J. Lumin. 1/2, 693 (1970).

${ }^{3}$ A. Sommerfeld, Partial Differential Equations in Physics (Academic, New York, 1949).

${ }^{4}$ R. Chance, A. Prock and R. Silbey, Adv. Chem. Phys. 37, 1 (1978).

${ }^{5}$ For the latest review, see W. L. Barnes, J. Mod. Opt. 45, 661 (1998).

${ }^{6}$ H. Benisty, R. Stanley, and M. Mayer, J. Opt. Soc. Am. A 15, 1192 (1998).

${ }^{7}$ I. Abram, I. Robert, and R. Kuszelewicz, IEEE J. Quantum Electron. 34, 71 (1998).

${ }^{8}$ R. L. Hartman, S. M. Cohen, and P. T. Leung (unpublished).

${ }^{9}$ Note that the $\delta$ function term is necessary for $G_{0}$ as explained in C. T. Tai, Proc. IEEE 61, 480 (1973).

${ }^{10}$ L. W. Li, J. A. Bennett, and P. L. Dyson, Int. J. Electron. 70, 803 (1991). 